\title{
Simultaneous Treatment with P53 Overexpression and Interferon $\gamma$ Exerts a Dramatic Increase in Apoptosis Induction of U87 Cells
}

Zahra Abbasy ${ }^{1}$, Hamid Zaferani Arani², Mahsa Ale-Ebrahim³, Vihan Moodi ${ }^{4}$ Javad Nematian² ${ }^{2}$ Mojdeh Barati ${ }^{5}$, Saba Shafaie $^{2}$, Alireza Madjid Ansari ${ }^{5}$, Atousa Hashemi ${ }^{6}$, Poorya Davoodi ${ }^{6}$, Mohammad Amin Javidi ${ }^{5 凶}$

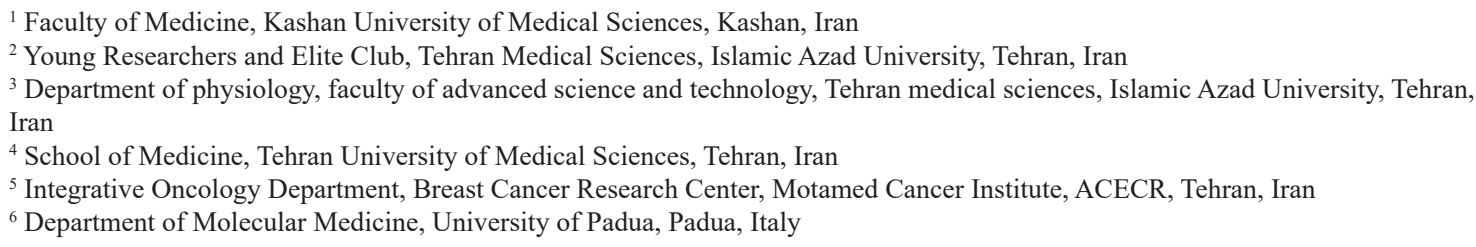

\begin{abstract}
Background: Gliomas possess low immunogenicity, which is an inevitable hinder in front of cancer immunotherapy. Different interferons (IFNs) may proceed apoptosis instead in p53-dependent or independent pathways. P53 induces the anti-inflammatory programmed cell death in cancer cells; on the other hand, IFN gamma (IFN $\gamma$ ) is a modulatory/proinflammatory cytokine. There are contradictory reports of whether this cytokine can possess an anti- or pro-cancerous impact on tumors. Hence, we aimed to investigate the possible cooperative apoptotic effect of the P53 and IFN $\gamma$ over expressions on the U87 glioblastoma cell line. Materials and Methods: The P53 expressing vector was amplified by Escherichia coli BL21. This vector was confirmed by the aid of sequencing. At the next step, U87 cells were transfected using lipofectamine. Cells were treated with P53 vector and/or IFN $\gamma$. The type of cellular death investigated by flow cytometry and the expression level of cleaved caspase-3 protein was also precisely demonstrated by western blotting. Results: Sequencing results revealed that inserted P53 was identical with human P53. Western blot results revealed that both IFN $\gamma$ and P53 overexpression could up-regulate cleaved caspase-3 protein expression in this cell line. Interestingly, flow cytometry data determined that concurrent treatment with P53 exogenous overexpression and IFN $\gamma$ induces about $70 \%$ apoptosis in U87; more than the sum of cell death occurs after IFN $\gamma$ or P53 overexpression alone $(\sim 18 \%+21 \%=39 \%)$. Conclusion: The present study results showed that $\mathrm{p} 53$-overexpression and IFN $\gamma$ could ultimately induce upregulation of the caspase- 3 and ultimately significant apoptosis increasing in the U87 cell line. Although IFN $\gamma$ is believed to be a pro-inflammatory cytokine and P53 is an anti-inflammatory agent, our results demonstrated that they could act synergistically to induce apoptosis in U87 cells.
\end{abstract}

[GMJ.2021;10:e2270] DOI:10.31661/gmj.v10i0.2270

Keywords: Glioblastoma; Caspase-3; Interferon $\gamma$; P53

\section{GMJ}

Copyright(C) 2021, Galen Medical Journal. This is an open-access article distributed under the terms of the Creative Commons Attribution 4.0 Internation License (http://creativecommons.org/licenses/by/4.0/) Email:info@gmj.ir $\bowtie$ Correspondence to:

Mohammad Amin Javidi, Integrative Oncology Department, Breast Cancer Research Center, Motamed Cancer Institute, ACECR, Tehran, Iran

Telephone Number:+98-9178376232

Email Address: maj136@gmail.com 


\section{Introduction}

Glioblastoma is the most aggressive and Uchallenging type of brain tumor; it is a high-grade astrocytoma that can develop in adults and children [1]. The median 5-year survival of malignant glioblastoma patients is about $2 \%$, and their overall survival is usually not more than one year and has not been improved significantly during the last three decades [1]. Meanwhile, 17000 patients are reported to be new cases each year in the United States, which is believed to uniformly result in mortality [2]. New approaches delineate a bright future in treating patients suffering glioblastoma; however, they substantially exert side effects and offer palliative outcomes rather than final cures [3]. It is important to notice that after introducing temozolomide as a new effective chemotherapy agent, adjuvant or concurrent prescription of it with chemotherapy or radiotherapy respectively, improved survival for just two months [2-5]. This highlights how critical it is to investigate new approaches for the treatment of this disease. One of the renowned compounds, which seems to play the role of Hecate for tumors, is a cytokine named interferon-gamma (IFN $\gamma$ ) [6]. IFN $\gamma$ is commonly secreted from natural immune cells, including T-cells found in the tumors' microenvironment and the tumor cells at low concentrations [7].

This master regulator can be beneficial in the immunotherapy of cancers with poor immunogenicity, as it is a pro-inflammatory cytokine and, according to the literature, upregulates major histocompatibility (MHC) class I [7].

At first glance, of course, this precious anti-cancer agent may have other sides and can be somehow believed to be a doubleedged sword, which confirms that the dark side should be investigated more deeply. By affecting fundamental characteristics of a tumor cell, IFN $\gamma$ has the potential to interfere with important tumorigenesis properties, including proliferation and angiogenesis; moreover, it can increase immunogenicity [6-8].

On the other hand, repeated vaccination with
IFN $\gamma$ may cause immunoediting in tumor cells, as reported in a study by Rearden et al. [9]. This phenomenon delineates double-face of IFN $\gamma$ for cancer treatment, which can cause resistance of tumor cells to the therapy, and even more emerges the importance of studies on the underlying pathways related to IFN $\gamma$ signaling in the tumor cells $[10,11]$.

P53 is a gatekeeper gene that acts as a tumor suppressor and is an obstacle against different cancer formation and development features, including angiogenesis inhibition, maintenance of genomic stability, etc. [10]. This famous anti-tumor gene is mutated in more than half of different cancers. Most of the tumors obtained from patients with glioblastoma multiform suffer deregulation of the P53 pathway [11]. Opposite to the IFN $\gamma$, P53 is an anti-inflammatory agent [12]. Based on the important anti-tumor role of the P53 and the high potential of the IFN $\gamma$ in apoptosis induction in tumor cells, this study aimed to assess the possible cooperative cytotoxic effect of both agents in a U87 glioblastoma cell line.

\section{Materials and Methods}

\section{Cell Culture}

U87 cells (purchased from Pasteur Institute, Iran) were cultured in high glucose dulbecco's modified eagle's medium (DMEM; Gibco, Germany) supplemented with $10 \%$ fetal bovine serum (FBS; Gibco, Germany) and 1\% penicillin-streptomycin as antibiotics (Gibco, Germany). IFN $\gamma$ was obtained from Sigma (Germany).

\section{Transformation and Plasmid Extraction} The pCMV-Neo-Bam-H1-p53 plasmid (P53 plasmid), expressing human wild-type P53, was kindly provided by Dr. Michael Resnick (NIEHS, NIH). P53 cDNA was inserted into the pCMV-NeoBam vector from Addgene (Cambridge, Massachusetts, USA), with the aid of cloning by the restriction enzyme BamHI (vector map is depicted in Figure-1). To amplify the plasmid, competent E. coli BL21 (Gibco, Germany) was transformed and cultured on Luria broth (LB) agar (Sigma, Germany) with $0.1 \%$ ampicillin (Gibco, Germany). 


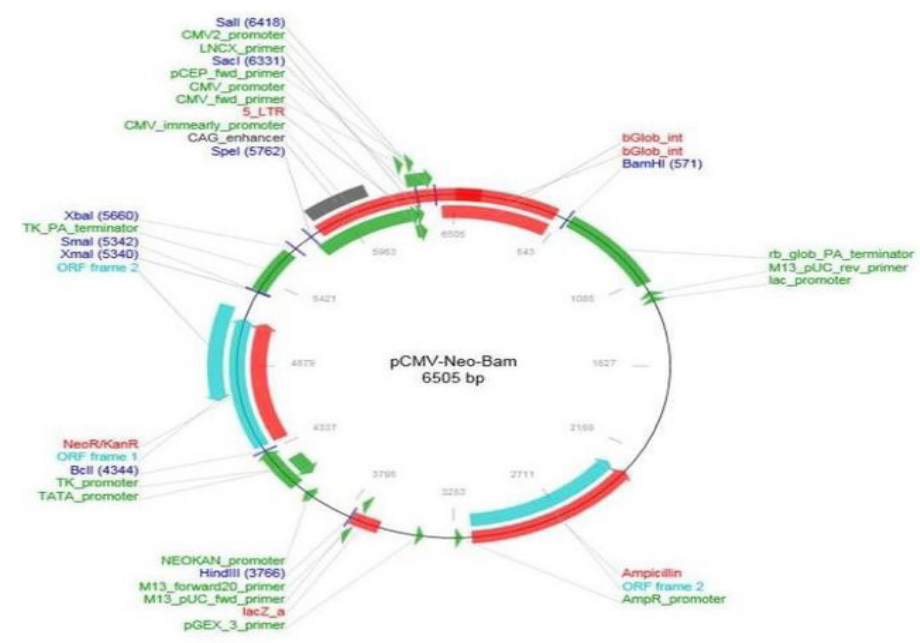

Figure 1. P53 plasmid map. The human P53 cDNA was cloned using the BamHI restriction enzyme (ampicillin-resistant gene was used as a selection marker for transformation).

To reach appropriate absorption, single colonies were isolated and cultured in LB medium.

Plasmids were isolated by BioNEER kit (South Korea, Figure-2).

\section{Transfection}

For transfection, Lipofectamine ${ }^{\circledR} 2000$ was utilized. Cells were seeded into 12-well plate 24 hours prior to the transfection without antibiotics; at the time of transfection, cells' confluency was about $70-80 \%$. The transfection was optimized, and the concentration of transfected plasmid was determined to be $100 \mathrm{ng} / \mu \mathrm{l}$ (we exploited a eukaryotic green fluorescent protein (GFP) containing plasmid to control the transfection). Plasmid with transfection reagents was incubated for $30 \mathrm{~min}$ to form plasmid:lipofectamine complex. This complex was added to each well after this time.

\section{Study Design}

Our main aim in this study was to determine whether P53 and IFN $\gamma$ act synergistically and exert anti-tumor activity on U87 cells; with this regard, we designed the experiment with four groups as follow:

1. Control group: untreated U87 cells that cultured in normal condition.

2. IFN $\gamma$ group: U87 cells that received IFN $\gamma$ $(100 \mathrm{ng} / \mathrm{ml})$ for 48 hours.

3. P53 group: U87 cells transfected with P53 plasmid according to the protocol mentioned above ( 72 hours).

4. $\mathrm{FN} \gamma+\mathrm{P} 53$ group: $\mathrm{U} 87$ cells transfected with the P53 plasmid and 24 hours later with 100 $\mathrm{ng} / \mathrm{ml}$ of IFN $\gamma$ (so the time P53 plasmid had 72 hours to be expressed, same time as the P53 group, and cells treated with IFN $\gamma$ for 48 hours, same as the IFN $\gamma$ group).

\section{Flow Cytometry}

To elaborately investigate whether cellular death induced post-treatment was necrosis or apoptosis, and to quantify apoptosis percent, Annexin V/PI (Roche, Switzerland) kit was performed, and cells' fluorescence was detected by flow cytometry device (FACScan flow cytometer, BD FacsCanto II, USA).

\section{Western Blotting}

Protein expression of the cleaved caspase- 3 was determined by western blotting in all groups. Cells were lysed, and the same amount of protein from each sample was utilized for this test. Firstly, proteins were separated by the aid of $10 \%$ SDS-PAGE and transferred to nitrocellulose membranes. These membranes were then incubated for 1 hour with 5\% nonfat milk at room temperature. Rabbit monoclonal antibodies against cleaved caspase- 3 proteins (Abcam, United Kingdom) were utilized as the primary antibody (membranes were incubated with primary antibody at $4^{\circ} \mathrm{C}$ overnight). A rabbit monoclonal antibody against $\beta$-actin 


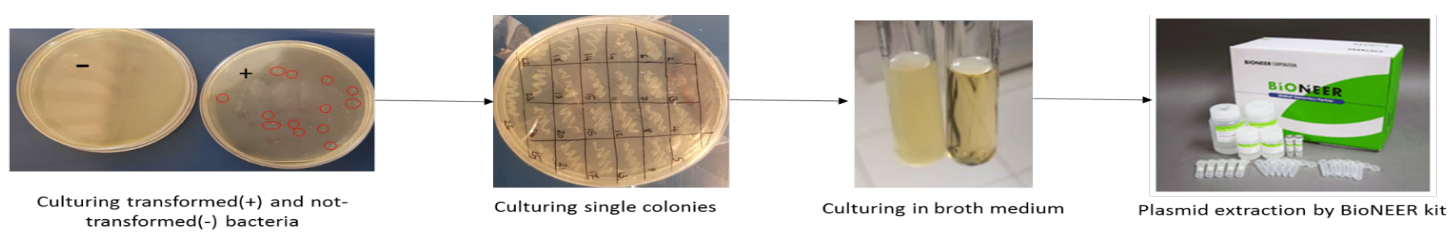

Figure 2. Plasmid transformation and extraction. Transformed bacteria were cultured on LB agar. After single colonies emerged, they were cultured separately on ampicillin-containing LB agar. They were then cultured in LB broth plus $0.1 \%$ ampicillin; after the optical density of the cultured bacteria became proper, plasmid extraction was performed.

was used as the control. Post washing step (washing buffer contained Tris-buffered saline and Tween 20), the membranes were incubated with the secondary antibody goat Anti-Rabbit IgG H\&L (HRP; Abcam, United Kingdom). The complexes were visualized with an Immobilon Western Chemiluminescent HRP substrate (Millipore, USA).

\section{Ethical Consideration}

This study has been approved by the ethics committee of Tehran Islamic Azad University of Medical Sciences (IR.IAU.TMU. REC.1399.373).

\section{Statistical Analysis}

All statistical analysis was accomplished by Graphpad Prism V8. For comparing whether differences between two and more samples were statistically significant, student $\mathrm{t}$-test and one-way ANOVA were carried out, respectively. Also, P-value less than 0.05 was considered as significant.

\section{Results}

\section{Plasmid Extraction}

Competent E. coli BL21 transformed for amplification of P53 plasmid. After transformation, single colonies (red circles in Figure-1) were isolated and cultured in LB broth medium containing $0.1 \%$ ampicillin as a selection marker.

After reaching proper optical density, plasmid extraction was performed with the BioNEER plasmid extraction kit (Figure-2).

\section{Vector Map and Sequencing}

To confirm the P53 sequence inserted in the plasmid, sequencing was performed for this cDNA with the universal primers of the vector. The inserted sequence was equal to the human P53 cDNA (Figure-3).

\section{Transfection Control}

To ensure that the transfection was performed functional, we utilized a eukaryotic GFP containing plasmid and transfected U87 cells with this plasmid as the same protocol used for the P53 plasmid. More than 70\% of transfected cells were GFP positive under a fluorescent microscope (Figure-4).

\section{Flow Cytometry}

Annexin V/PI flow cytometry results for different samples implicated that the IFN $\gamma$ group had about $18 \%$ cell death (100-cell viability), and the P53group had about $21 \%$ cell death. Interestingly when applied together in the P53+IFN $\gamma$ group, cell death was calculated to be about 70\% (Figure-5).

\section{Western Blotting}

The expression level of cleaved caspase-3 protein, as an execution caspase and related downstream of both Bak and Bax up-regulationwhich can be induced by IFN $\gamma$ and p53, respectively-determined by western blotting.

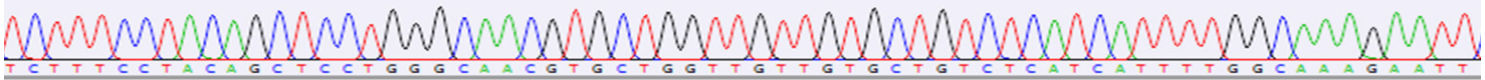

Figure 3. Sequencing results revealed that cloned P53 was identical with the human P53 cDNA sequence. 


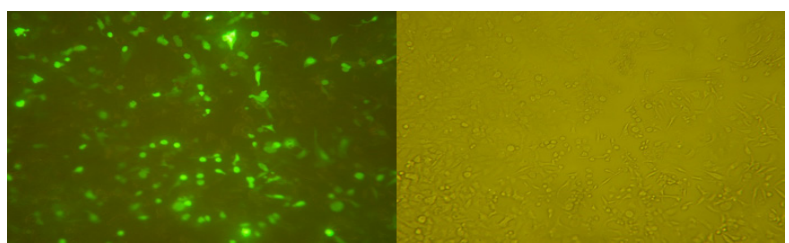

Figure 4. Transfection control by eukaryotic GFP containing the plasmid. About $70 \%$ of transfected cells were GFP positive after transfection under a fluorescent microscope (magnification 400x).
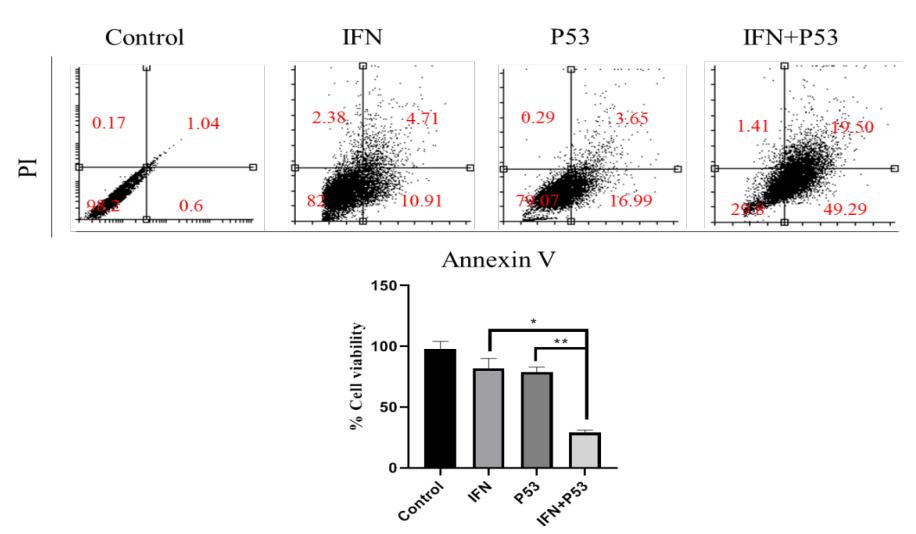

Figure 5. Data obtained from Annexin V/PI flow cytometry indicates that treatment of U87 cells with IFNy induces about $18 \%$ cell apoptosis. Similarly, when these cells were transfected with P53 overexpressing vector, about $21 \%$ apoptosis occurred in the transfected cells. In the concurrent treatment of U87 cells with IFNy and the mentioned vector, about $70 \%$ apoptosis was induced in the treated cells.

\section{$\beta$-actin}
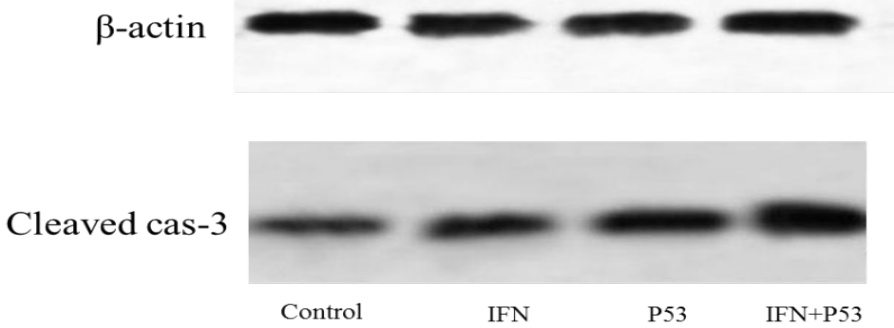

Figure 6. Western blotting data revealed that cleaved caspase-3 protein level was much higher in the P53+IFNy group than in other groups.

As it is shown in Figure-5, the protein level of the cleaved caspase- 3 normalized to the internal protein $\beta$-actin increased in treated groups, including the U87 cells that were transfected with a P53-overexpressed plasmid as well as groups treated with IFN $\gamma$ or concurrent treatment with both (IFN $\gamma$ and P53-overexpressed plasmid).

Furthermore, this expression level was much higher in the P53+IFN $\gamma$ group than in the other groups (Figure-6).

\section{Discussion}

High-grade astrocytoma is called glioblastoma, which is the most malignant of all brain tumors and accounts for the majority of gliomas [1]. Glioblastoma symptoms are subtle at first, and some types of gliomas do not cause any symptoms, which results in high mortality and calls for more demand of studies at detection and/or treatment steps [12, 13]. Gliomas are low immunogenic, which obstruct 
immunotherapy. With this regard, different studies performed struggle to increase immunogenicity in these tumors [14].

IFN $\gamma$ is a renowned master regulator and possesses pro-inflammatory characteristics that can up-regulate MHC class I and increase immunogenicity; however, its cytotoxicity does not fulfill for treatment of tumors; mainly pieces of evidence exist that in repeated vaccination of IFN $\gamma$ in NHL mice models, it can act reversely and causes tumor resistance $[8,9,11]$.

With this regard, we exploited IFN $\gamma$ treatment in a U87 cell line. Our study revealed IFN $\gamma$ treatment alone caused about $20 \%$ of cell death, which was not satisfactory at first glance. For increasing the efficiency of the treatment, we intended to investigate whether overexpression of P53 can elevate the apoptosis rate in these cells. Hence, at the next step, we assessed the possible synergistic apoptotic outcome of the IFN $\gamma$ and P53 overexpression on U87 cells. Interestingly, we found out when treating merely with just IFN $\gamma$ or overexpressing P53, about $20 \%$ of cell death occurs; and in cells treated with both, the rate of cellular death increased up to about $80 \%$. In the following steps, we tried to shed light on the possible joint molecular mechanism of action of these two treatments. Qian et al. [15] assessed the role of IFN $\gamma$ in immune evasion of orthologous glioma tumors in mice models. They concluded that IFN $\gamma$ is important in the up-regulation of PD-L1 (an immune suppression receptor that was expressed on the surface of many cancer cells and by interaction with PD1 causes dysfunction of T-cells) even in the tumor microenvironment and act as tumor progressive agent. Furthermore, it is reported that $\mathrm{CD}^{8+}$ T-cells secret IFN $\gamma$ near the tumor sites, sequentially affecting the JAK/ STAT pathway and IRF-1 leading to the upregulation of PD-L1 in cancer cells [15].

Chemotherapy agents may induce apoptosis with two somehow related predominant pathways, including extrinsic and intrinsic [16].

In the extrinsic pathways, death receptors like the tumor necrosis factor (TNF) family, DR4, DR5, and CD95/APO-1/Fas ligands play important roles [17]. When stimulated, they cause conversion of inactive pro-caspases- 8 and -10 into active initiator, which can envisage activation of Bid (a pro-apoptotic Bcl2 family protein) and release of cytochrome $\mathrm{C}$ from mitochondria into the cytoplasm [17]. In the intrinsic pathway, mitochondria play a crucial role; and after stimulation, cytochrome $\mathrm{C}$, Apaf-1, and caspase-9 are released to the cytoplasm, where the association of calcium ions forms apoptosome and in turn results in active caspase-3 [16-21]. Similarly, our western blot results revealed that after

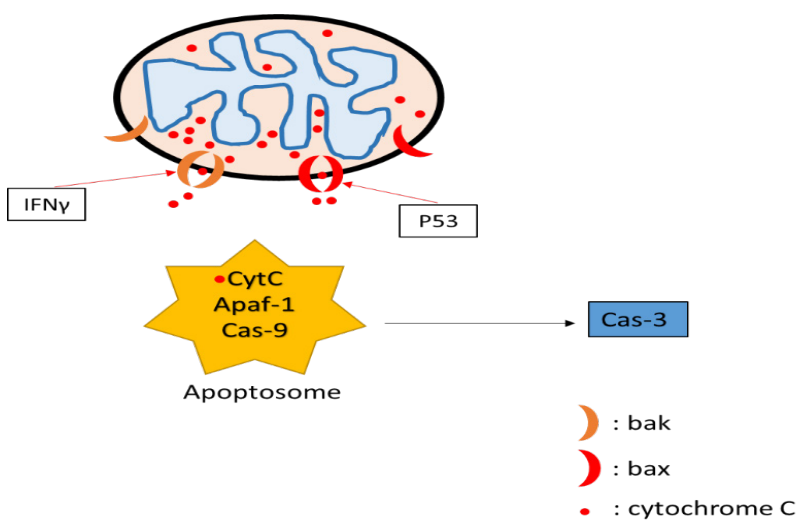

Figure 7. The schematic figure for a possible relationship between IFNy and P53 apoptosis induction. IFNy can induce the oligomerization of the Bak proteins (a pro-apoptotic member of the bcl-2 family), P53 can induce the oligomerization of the Bax proteins (another pro-apoptotic bcl-2 family member). Both the oligomerized Bak and Bax can induce the permeabilization of the mitochondrial outer membrane (MOM). This leads to the release of cytochrome $\mathrm{C}$ into the cytoplasm. The cytoplasm cooperates with other proteins (e.g., Apaf-1 and caspase-9) to produce an apoptosome complex. Apoptosome can induce the pro-caspase-3 into the cleaved/activated form of the caspase-3. This caspase is an execution caspase and can trigger other caspases (e.g., 6 and 7 ) activation and lead cell towards apoptosis. 
treatment of U87 cells with IFN $\gamma$ and/or P53 overexpression, the expression level of cleaved caspase- 3 increased (this may be a reliable joint venture between these two treatments). Tekautz et al. [22] demonstrated that IFN $\gamma$ induces up-regulation of caspase-8 (an essential initiator caspase in the extrinsic pathway) in neuroblastoma cell lines [22]. Similarly, Afshar et al. [23] in gliomas cell lines without wild-type P53, radiation induces caspase-8 dependent, P53 independent apoptosis [23]. According to the literature, the U87 cells that possess wild-type P53 are more resistant to radiotherapy $[23,24]$. Our results indicate that overexpression of P53 in the U87 cell line can potentially sensitive it to treatments.

On the other hand, IFN $\gamma$ can induce different pro-apoptotic pathways in cancer cells [25-34]. A study performed by Ruiz-Ruiz et al. [35] demonstrated that IFN $\gamma$ sensitive breast cancer cells to death regardless of P53 status by activating CD95 death receptor, up-regulation of caspase- 8 , and release of cytochrome $\mathrm{C}$ from mitochondria. In the current study, we assessed whether overexpression of P53 can synergistically act with IFN $\gamma$ in the U87 cell line. Interestingly, flow cytometry data determined that concurrent treatment with P53 exogenous overexpression and IFN $\gamma$ induces about $70 \%$ apoptosis in U87 cells; more than the sum of cell death occurs after IFN $\gamma$ or P53 overexpression alone (approximately 39\%).

Regarding western blotting results, the U87 cells that received IFN $\gamma$ and P53, the protein expression level of the cleaved caspase- 3 was higher than the untreated control or other groups, which received merely IFN $\gamma$ or $\mathrm{p} 53$ overexpression treatment alone. Interestingly, our results confirm that both IFN $\gamma$ and P53 can lead to the same cell destiny, while the direct targets of them were different.

IFN $\gamma$ does not induce Bax, a pro-apoptotic target for P53 [36].

Bak and Bax are the building blocks that can permeabilize mitochondrial outer membrane (MOM) by oligomerization [36-40]. However, both two compounds (IFN $\gamma$ and p53) could induce a common pathway of cytochrome $\mathrm{C}$ release from MOM into the cytoplasm [38].

Consequently, assembly of apoptosome complex with the aid of Apaf-1 and caspase-9, which results in activation (cleavage) of the execution caspase, caspase- 3 , and finally caspase cascade (Figure-7) [40].

\section{Conclusions}

According to our results, IFN $\gamma$ and P53 can act cooperatively to induce apoptosis in U87 cells with the aid of caspase-3 activation. Hence simultaneous treatment with both IFN $\gamma$ and P53 can be exploited to elevate the treatment efficiency, especially in cancers with wild-type P53. However, complementary investigations are an obvious demand prior to the instigation of the bench to the bedside.

\section{Conflict of Interest}

The authors declare that there are no conflicts of interest.

\section{References}

1. Ansari M, Nasrolahi H, Kani AA, Mohammadianpanah M, Ahmadloo N, Omidvari S, et al. Pediatric glioblastoma multiforme: A single-institution experience. Indian J Med Paediatr Oncol. 2012;33(3):155.

2. Hosseini MM, Karimi A, Behroozaghdam M, Javidi MA, Ghiasvand S, Bereimipour A, Aryan H, Nassiri F, Jangholi E. Cytotoxic and apoptogenic effects of cyanidin-3-glucoside on the glioblastoma cell line. World neurosurgery. 2017;108:94-100.

3. Wang G, Wang JJ, Fu XL, Guang R, To SS. Advances in the targeting of HIF$1 \alpha$ and future therapeutic strategies for glioblastoma multiforme. Oncol Rep. 2017;37(2):657-70.

4. Chen J, Xu T. Recent therapeutic advances and insights of recurrent glioblastoma 
multiforme. Front Biosci (Landmark Ed). 2013;18:676-84.

5. Robins HI, Chang S, Butowski N, Mehta M. Therapeutic advances for glioblastoma multiforme: current status and future prospects. Curr Oncol Rep. 2007;9(1):6670.

6. Schroder K, Hertzog PJ, Ravasi T, Hume DA. Interferon- $\gamma$ : an overview of signals, mechanisms and functions. $\mathrm{J}$ Leukoc Biol. 2004;75(2):163-89.

7. Kelderman S, Schumacher TN, Haanen JB. Acquired and intrinsic resistance in cancer immunotherapy. Mol Oncol. 2014;8(6):1132-9.

8. Zaidi MR, Merlino G. The two faces of interferon- $\gamma$ in cancer. Clin Cancer Res. 2011;17(19):6118-24.

9. Rearden R, Sah A, Doff B, Kobayashi T, McKee SJ, Leggatt GR, et al. Control of B-cell lymphoma by therapeutic vaccination and acquisition of immune resistance is independent of direct tumour IFN-gamma signalling. Immunol Cell Biol. 2016;94(6):554-62.

10. Mattarollo SR, West AC, Steegh K, Duret H, Paget C, Martin B, et al. NKT cell adjuvant-based tumor vaccine for treatment of myc oncogene-driven mouse B-cell lymphoma. Blood, The Journal of the American Society of Hematology. 2012;120(15):3019-29.

11. John LB, Darcy PK. The double-edged sword of IFN-[gamma]-dependent immune-based therapies. Immunol Cell Biol. 2016;94(6):527.

12. Newton HB. Molecular neuro-oncology and the development of targeted therapeutic strategies for brain tumors. Part 3: brain tumor invasiveness. Expert Rev Anticancer Ther. 2004;4(5):803-21.

13. Weller M, Wick W, Aldape K, Brada M, Berger M, Pfister SM, et al. Glioma. Nat Rev Dis Primers. 2015;1(1):1-8., 2015.

14. Guan X, Hasan MN, Begum G, Kohanbash G, Carney KE, Pigott VM, et al. Blockade of $\mathrm{Na} / \mathrm{H}$ exchanger stimulates glioma tumor immunogenicity and enhances combinatorial TMZ and anti-PD-1 therapy. Cell Death Dis. 2018;9(10):1-6.

15. Qian J, Wang C, Wang B, Yang J, Wang Y, Luo F, et al. The IFN- $\gamma /$ PD-L1 axis between
T cells and tumor microenvironment: hints for glioma anti-PD-1/PD-L1 therapy. J neuroinflammation. 2018;15(1):1-3.

16. Gul-e-Saba Chaudhry RJ, Zafar MN, Mohammad H, Muhammad TS. Vitex rotundifolia fractions induced apoptosis in human breast cancer T-47D cell line via activation of extrinsic and intrinsic pathway. Asian Pac J Cancer Prev. 2019;20(12):3555.

17. Rachakhom W, Khaw-On P, Pompimon W, Banjerdpongchai R. Dihydrochalcone derivative induces breast cancer cell apoptosis via intrinsic, extrinsic, and ER stress pathways but abolishes EGFR/ MAPK pathway. Biomed Res Int. 2019;2019.

18. Lu Z, Zhou H, Zhang S, Dai W, Zhang $\mathrm{Y}$, Hong L, et al. Activation of reactive oxygen species-mediated mitogenactivated protein kinases pathway regulates both extrinsic and intrinsic apoptosis induced by arctigenin in Hep G2. J Pharm Pharmacol. 2020;72(1):2943.

19. Engels IH, Stepczynska A, Stroh C, Lauber K, Berg C, Schwenzer R, et al. Caspase-8/FLICE functions as an executioner caspase in anti-cancer drug-induced apoptosis. Oncogene. 2000;19(40):4563-73.

20. Tekautz T, Teitz T, Lahti JM, Kidd VJ. Proapoptotic Gene Silencing Via Methylation in Human Tumors. InDeath Receptors in Cancer Therapy 2005 (pp. 207-229). Humana Press.

21. Reed JC, Tomaselli KJ. Drug discovery opportunities from apoptosis research. Curr Opin Biotechnol. 2000;11(6):58692.

22. Tekautz TM, Zhu K, Grenet J, Kaushal D, Kidd VJ, Lahti JM. Evaluation of IFN- $\gamma$ effects on apoptosis and gene expression in neuroblastoma-preclinical studies. Biochim Biophys Acta. 2006;1763(10):1000-10.

23. Afshar G, Jelluma N, Yang X, Basila D, Arvold ND, Karlsson A, et al. Radiationinduced caspase- 8 mediates p53independent apoptosis in glioma cells. Cancer Res. 2006;66(8):4223-32.

24. Cerrato JA, Yung WA, Liu TJ. Introduction 
of mutant p53 into a wild-type p53expressing glioma cell line confers sensitivity to Ad-p53-induced apoptosis. Neuro-oncology. 2001 Apr 1;3(2):113-22.

25. Li L, Chen SN, Laghari ZA, Huang B, Huo HJ, Li N, et al. Receptor complex and signalling pathway of the two type II IFNs, IFN- $\gamma$ and IFN- $\gamma$ rel in mandarin fish or the so-called Chinese perch Siniperca chuatsi. Dev Comp Immunol. 2019;97:98-112.

26. Dos Santos RS, Marroqui L, Velayos T, Olazagoitia-Garmendia A, JauregiMiguel A, Castellanos-Rubio A, et al. DEXI, a candidate gene for type 1 diabetes, modulates rat and human pancreatic beta cell inflammation via regulation of the type I IFN/STAT signalling pathway. Diabetologia. 2019;62(3):459-72.

27. Chen S, Wu Z, Zhang J, Wang M, Jia R, Zhu D, et al. Duck stimulator of interferon genes plays an important role in host anti-duck plague virus infection through an IFN-dependent signalling pathway. Cytokine. 2018;102:191-9.

28. Fleming SB. Viral inhibition of the IFNinduced JAK/STAT signalling pathway: development of live attenuated vaccines by mutation of viral-encoded IFNantagonists. Vaccines. 2016;4(3):23.

29. Park GB, Hur DY, Kim YS, Lee HK, Yang JW, Kim D. TLR 3/TRIF signalling pathway regulates IL-32 and IFN- $\beta$ secretion through activation of RIP-1 and TRAF in the human cornea. J Cell Mol Med. 2015 May;19(5):1042-54.

30. Chow YL, Lee KH, Vidyadaran $\mathrm{S}$, Lajis NH, Akhtar MN, Israf DA, et al. Cardamonin from Alpinia rafflesiana inhibits inflammatory responses in IFN- $\gamma /$ LPS-stimulated BV2 microglia via NF- $\kappa \mathrm{B}$ signalling pathway. Int Immunopharmacol. 2012;12(4):657-65.

31. Bambard ND, Mathew SO, Mathew PA. LLT1-mediated Activation of IFN- $\gamma$ Production in Human Natural Killer Cells Involves ERK Signalling Pathway. Scand J Immunol. 2010;71(3):210-9.

32. Wei B, Baker S, Wieckiewicz J, Wood KJ. IFN- $\gamma$ triggered STAT1-PKB/AKT signalling pathway influences the function of alloantigen reactive regulatory $\mathrm{T}$ cells.
Am J Transplant. 2010 Jan;10(1):69-80.

33. Liu LD, Dong CH, Shi HJ, Zhao HL, Wang LC, Ma SH, et al. A novel type II membrane receptor up-regulated by IFN- $\alpha$ in fibroblasts functions in cell proliferation through the JAKSTAT signalling pathway. Cell prolif. 2006;39(2):93-103.

34. Imaizumi T, Kumagai M, Taima K, Fujita T, Yoshida H, Satoh K. Involvement of retinoic acid-inducible gene-I in the IFN- $\gamma /$ STAT1 signalling pathway in BEAS-2B cells. Eur Respir J. 2005;25(6):1077-83.

35. Ruiz-Ruiz C, Muñoz-Pinedo C, LópezRivas A. Interferon- $\gamma$ treatment elevates caspase- 8 expression and sensitizes human breast tumor cells to a death receptorinduced mitochondria-operated apoptotic program. Cancer Res. 2000;60(20):567380.

36. Westphal D, Kluck RM, Dewson G. Building blocks of the apoptotic pore: how Bax and Bak are activated and oligomerize during apoptosis. Cell Death Differ. 2014;21(2):196-205.

37. Luna-Vargas MP,ChipukJE. Physiological and pharmacological control of BAK, BAX, and beyond. Trends Cell Biol. 2016;26(12):906-17.

38. Peña-Blanco A, García-Sáez AJ. Bax, Bak and beyond - mitochondrial performance in apoptosis. FEBS J. 2018;285(3):41631.

39. Ossina NK, Cannas A, Powers VC, Fitzpatrick PA, Knight JD, Gilbert JR, et al. Interferon- $\gamma$ modulates a p53independent apoptotic pathway and apoptosis-related gene expression. J Biol Chem. 1997;272(26):16351-7.

40. Castro F, Cardoso AP, Gonçalves RM, Serre K, Oliveira MJ. Interferon-gamma at the crossroads of tumor immune surveillance or evasion. Front Immunol. 2018;9:847. 